\title{
ESTUDO DE UTILIZAÇÃO DE RETINOIDES SISTÊMICOS: UMA ANÁLISE DAS NOTIFICAÇÕES DE RECEITA ESPECIAIS ENTREGUES A VIGILÂNCIA SANITÁRIA DE MUNICÍPIO DA GRANDE RECIFE
}

\author{
STUDY USE OF SYSTEMIC RETINOIDS: AN ANALYSIS OF SPECIAL \\ REVENUE NOTIFICATIONS DELIVERED TO THE SANITARY \\ SURVEILLANCE OF METROPOLITAN RECIFE'S CITY REGION
}

\author{
Samantha Mayara de Sousa Silva ${ }^{1}$ \\ Clarissa Oliveira Lima Silva ${ }^{2}$ \\ Adriana Amorim de Farias Leal ${ }^{3}$ \\ Rômulo Moreira dos Santos ${ }^{4}$
}

RESUMO: OBJETIVO: Examinar as Notificações de Receita Especiais e Termos de Conhecimento de Riscos e Consentimento Pós-Informação entregues a uma vigilância sanitária, utilizados para prescrição e dispensação de medicamentos contendo retinoides sistêmicos. MÉTODO: O desenho do estudo foi de cunho transversal, com abordagem quantitativa, sendo realizado no Município de Goiana, situado na Região Metropolitana do Recife, Pernambuco, durante os meses de junho a dezembro de 2019. Para tanto, observou-se os campos prescricionais da notificação de receita especial, em cumprimentos as normas legais, a legibilidade do manuscrito e preenchimento dostermos de consentimento, só após a aprovação por comitê de ética, recebendo a CAAE $n^{\circ}$ 11673219.7.0000.5175. RESULTADOS: Foram analisadas cem (100) notificações de receita especiais (NRR) dispensadas no período entre junho de 2018 a junho de 2019, sendo em sua totalidade para prescrição da isotretinoína, sendo as usuárias do sexo feminino as mais prevalentes. Quanto a legibilidade, em sua maioria $(82,30 \%)$ as receitas foram consideradas legíveis, porém, alguns parâmetros avaliados foram classificados como parcialmente legíveis ou ilegíveis. CONCLUSÃO: Apesar das orientações sanitárias serem claras quanto às exigências para prescrição e dispensação destes medicamentos sujeitos à controle especial, ainda há transgressões às normas sanitárias estabelecidas e práticas que podem induzir aos erros de medicação, comprometendo a segurança

\footnotetext{
${ }^{1}$ Graduanda do curso de Farmácia da UNIFACISA.

${ }^{2}$ Graduanda do curso de Farmácia da UNIFACISA.

${ }^{3}$ Farmacêutica e Estudante do Programa de Pós-Graduação em Saúde Coletiva da UFRN.

4 Farmacêutico, Mestre em Saúde Pública e Professor dos cursos de Farmácia e Nutrição da UNIFACISA.
} 
do usuário dos medicamentos, como a existência de ilegibilidade de informações da notificação de receita. É preciso ainda mais informações para promoção da educação em saúde quanto a essas práticas e atividades de fomento ao uso racional dos medicamentos.

Palavras chave: Notificação de Receita. Isotretinoína. Farmacoepidemiologia.

ABSTRACT: OBJECTIVE: To examine the Special Prescription Notifications and Post-Information Consent Risk and Consent Knowledge Terms delivered to health surveillance, used for prescription and dispensing of medications containing systemic retinoids. METHOD: The study design was cross- sectional, with a quantitative approach, being carried out in the Goiana city, located in the Metropolitan Region of Recife, Pernambuco, during the months of june to december 2019. Therefore, the prescriptive fields were observed the notification of special prescription, in compliance with legal norms, the legibility of the manuscript and filling in the terms of consent, only after approval by the ethics committee, receiving CAAE $n^{\circ}$ 11673219.7.0000.5175. RESULTS: One hundred (100) special prescription notifications (NRR) dispensed in the period between June 2018 to June 2019 were analyzed, being in its entirety for the prescription of isotretinoin, with female users being the most prevalent. As for legibility, the majority (82.30\%) of the recipes were considered legible, however, some parameters evaluated were classified as partially legible or illegible. CONCLUSION: Although the health guidelines are clear about the requirements for prescribing and dispensing these drugs subject to special control, there are still violations of established health rules and practices that can lead to medication errors, compromising the safety of the user of the drugs, such as the existence of illegibility of information in the revenue notification. Even more information is needed to promote health education regarding these practices and activities to promote the rational use of medicines.

Keywords: Drug Prescription of Special Control. Isotretinoin. Pharmacoepidemiology. 


\section{INTRODUÇÃO}

Atualmente, dentre as inúmeras apresentações farmacêuticas, encontram-se substânciascapazes de moldar funções fisiológicas humanas que exercem alterações comportamentais e no estilo de vida do paciente que as utiliza. Além das substâncias de controle especial por apresentarem ação no sistema nervoso central, os fármacos com reconhecidos risco e gravidade aos usuários, também são regulados por documentos legais em muitos países. Esse é o caso dos retinoides sistêmicos, que apresentam efeitos teratogênicos mesmo se usados por um curto período de tempo (VALLERAND et al., 2018).

Na Lei Federal n 5.991 de 1973 (BRASIL, 1973) que dispõe sobre o controle sanitáriodo comércio de drogas, medicamentos, insumos farmacêuticos e correlatos, constam as primeiras normativas para o gerenciamento dos produtos sujeitos a controle especial, como a comprovação em balanços de venda e dispensação.

Com a Portaria SVS 344/1998 (BRASIL, 1998) e suas atualizações, a Secretaria de Vigilância Sanitária emitiu regras mais detalhadas para a produção, gerenciamento e comercialização destes medicamentos, na qual há uma forma de prescrição para cada classe demedicamento abrangido nos anexos deste dispositivo legal. No caso dos retinoides de uso sistêmico, faz-se necessária a prescrição por profissional médico da Notificação de Receita Especial (NRR) e do Termo de Conhecimento de Riscos e Consentimento Pós-Informação, paratodos os usuários.

Ainda, segundo a Portaria 06/1999 (BRASIL, 1999), que aprovou o Regulamento Técnico das substâncias e medicamentos sujeitos a controle especial presentes na Portaria 344/1998, há o reforço de normas sanitárias, como a exigência do peticionamento de autorização às vigilâncias sanitárias competentes para comercialização destes medicamentos, além da entrega de cópias de todas as NRR e termos de consentimento anexados aos Balanços de Medicamentos Psicotrópicos e Outras substâncias (BMPO), trimestralmente a estes órgãos de fiscalização. 
Tais notificações, já possuem validade nacional para facilitar o tratamento de usuários em trânsito dentro do território nacional, de acordo com a Lei Federal 13.732/2018, que alterou o texto do parágrafo único do artigo 35 , presente na Lei Federal 5.991/73, ainda vigente (BRASIL, 1973; BRASIL, 2018).

Desde esta última legislação citada, já se exigia que as prescrições medicamentosas fossem completamente legíveis e não poderiam apresentar rasuras, siglas ou emendas que comprometessem a interpretação do medicamento prescrito e informações dos usuários contidas nos documentos.

O controle sanitário destes medicamentos se faz importante para cumprimento da legislação pertinente e propiciar as análises de uso destes produtos no âmbito farmacoepidemiológico e conhecimento dos possíveis problemas sanitários e riscos à saúde dosusuários.

\section{MÉTODOS}

O desenho do estudo foi de cunho transversal, com abordagem quantitativa, sendo realizado no Município de Goiana, situado na Região Metropolitana do Recife, Pernambuco, durante os meses de junho a dezembro de 2019.

Para a pesquisa, foram usados como fonte de dados os BMPO, contendo as cópias das NRR, entregues trimestralmente a Vigilância Sanitária municipal e com datas de prescrição entre junho de 2018 a junho de 2019. A coleta de dados foi realizada no Departamento de Vigilância Sanitária do município citado após o recebimento e conferência dos documentos, utilizando-se um formulário padrão para transcrição das informações referentes aos campos prescricionais da NR com informações do prescritor, paciente, medicamento e da legibilidade do manuscrito, conforme a classificação proposta por Rosa et al. (2009), onde cada palavra deveria ser avaliada separadamente, evitando interpretação ou dedução, assim, a prescrição deveria ser entendida e não interpretada, classificando-as como Legível (leitura fácil, sem problema ou perda de tempo para compreensão do que está escrito ou com dúvidas para o entendimento de todas as palavras, siglas e números), Parcialmente 
legível (leitura com dificuldade, utilizando-se um maior tempo para compreensão do manuscrito) e llegível (quando, independentemente do tempo da análise, foi impossível de entender o que estava escrito).

O estudo obedeceu a Resolução n 466/2012 do Conselho Nacional de Saúde e só foi desenvolvido após a aprovação pelo Comitê de Ética em Pesquisa do Centro de Ensino Superior e Desenvolvimento (CESED), recebendo a CAAE $n^{\circ}$ 11673219.7.0000.5175.

\section{RESULTADOS}

Foram analisadas cem (100) NRR dispensadas no período do estudo, sendo em sua totalidade para prescrição da isotretinoína e o sexo feminino foi o mais prevalente, representando mais da metade dos usuários da amostra analisada. Após aplicabilidade do formulário padrão e análise das NRR, os resultados obtidos estão descritos na Tabela1.

TABELA 1. DISTRIBUIÇÃO PERCENTUAL DAS VARIÁVEIS PRESCRICIONAIS DAS NR E DADOS DOS TERMOS DE CONSENTIMENTO AVALIADOS PELO ESTUDO.

\begin{tabular}{lcc}
\hline Variáveis & N & \% \\
\hline Sexo $\quad$ Feminino & 54 & 54,00 \\
$\quad$ Masculino & 46 & 46,00 \\
Procedência do Paciente & & \\
$\quad$ Pernambuco & 97 & 97,00 \\
$\quad$ Outro Estado & 03 & 03,00 \\
NR própria do prescritor & & \\
$\quad$ Sim & 41 & 41,00 \\
$\quad$ Não & 59 & 59,00 \\
Especialidade Médica Dermatologista & & 85,00 \\
$\quad$ Clínico Geral & 85 & 15,00 \\
$\quad$ Medicamento e Dose & 15 & 100,00 \\
$\quad$ Isotretinoína 20mg & 100 & 25,00 \\
Posologia & &
\end{tabular}


2 comprimidos ao dia

3 comprimidos ao dia

Uso de anticoncepcional

Sim

Não

Dispensação Farmacêutica

Sim

Não

Medicamento e Dose

Isotretinoína $20 \mathrm{mg}$

Quantidade Dispensada

1 caixa

2 caixas

3 caixas

Tipo Referência

Genérico

Identificação do laboratório

Sim

Não

Presença de Lote

Sim

Não
72

03

38

62

12

88

100

24

73

03

02

98

79

21

94

06
72,00

03,00

38,00

62,00

12,00

88,00

100,00

24,00

73,00

3,00

2,00

98,00

79,00

21,00

TOTAL

100

94,00

06,00

Fonte: dados do estudo, 2019.

NR = Notificação de Receita.

De modo geral, os dados obtidos quanto ao que se refere a legibilidade das Notificaçõesde Receitas, estão descritos na Tabela 2:

TABELA 2. ANÁLISE QUANTO À LEGIBILIDADE DAS NRR.

\begin{tabular}{lcc}
\hline Variáveis & $\mathbf{N}$ & $\%$ \\
\hline Dados do Paciente & 65 & 65,00 \\
$\quad$ Legível & 32 & 32,00 \\
$\quad$ Parcialmente legível & 3 & 3,00 \\
$\quad$ llegível & & \\
Dados do Prescritor & 88 & 88,00 \\
$\quad$ Legível & 10 & 10,00 \\
$\quad$ Parcialmente legível & 2 & 2,00 \\
$\quad$ llegível & & \\
Nome do Medicamento Legível & 95 & 95,00 \\
$\quad$ Legível & 4 & 4,00 \\
$\quad$ Parcialmente legível & 1 & 1,00 \\
$\quad$ llegível & &
\end{tabular}




\begin{tabular}{lcc} 
Legível & 92 & 92,00 \\
Parcialmente legível & 7 & 7,00 \\
llegível & 1 & 1,00 \\
Posologia & 76 & 76,00 \\
Legível & 23 & 23,00 \\
Parcialmente legível & 1 & 1,00 \\
\hline Ilegível & $\mathbf{1 0 0}$ & $\mathbf{1 0 0 , 0 0}$ \\
\hline TOTAL & &
\end{tabular}

Fonte: dados do estudo, 2019.

NRR = Notificação de Receita Especial.

\section{DISCUSSÃO}

As NRR direcionadas aos retinoides sistêmicos podem ser utilizadas para compra dos medicamentos prescritos em até 30 dias a contar da data nas quais foram prescritas e, habitualmente, podem conter tratamento necessário para 30 dias (BRASIL, 1998). Com relação a esta exigência, não foram encontradas NRR que transgrediram a norma, nem pelas informações da prescrição ou pelo registro de venda informado pelos BMPO das farmácias consultadas.

É importante saber que, de acordo com o protocolo de uso da Isotretinoína, a alteração no perfil lipídico é um efeito colateral comum de uso (BRASIL, 2015). Em um estudo de coorte populacional foi mostrado que a elevação de triglicerídeos ocorreu em $45 \%$ dos pacientes durante o tratamento e que o aumento de colesterol total foi encontrado em $30 \%$ deles (ZANEet al., 2006). Geralmente as elevações são leves e não determinam a interrupção do tratamento. No caso de alteração do perfil lipídico, os pacientes devem ser seguidos do ponto de vista clínico e laboratorial a cada 3 meses (BRASIL, 2015). Como esta informação não é uma exigência para a prescrição ou dispensação de medicamentos com esta substância, pode- se considerar uma falha do ponto de vista clínico e prejudicial ao uso racional de medicamentos. Para cada NRR, existe uma numeração única capaz de conferir o controle para a vigilância sanitária competente, que trimestralmente recebe estas 
NR dos medicamentos dispensados, após serem devidamente registradas ao SNGPC (Sistema Nacional de Gerenciamento de Produtos Controlados).

É importante frisar que de acordo com a Constituição da República Feerativa do Brasilde 1988 (BRASIL, 1988) e do Código de Defesa do Consumidor na Lei n. 8.078, de 1990 (BRASIL, 1990), o médico não pode submeter o seu paciente a tratamento ou procedimento terapêutico sem antes obter seu consentimento.

Para tanto, as normas de controle e comercialização dos medicamentos e substâncias sujeitas à controle especial exigem, além da NR específica, o Termo de Consentimento Pós- Informação", que registra a decisão pacífica entre o paciente e profissional prescritor, com base no risco-benefício do tratamento e sabendo dos potenciais efeitos colaterais relacionados ao uso do medicamento retinoide sistêmico. O Termo de Consentimento é obrigatório ao se prescreverestes medicamentos que também fazem parte das opções de Componentes Especializados da Assistência Farmacêutica (BRASIL, 1990).

No preenchimento deste termo, há informações específicas para as pacientes do sexo feminino, pois devido ao risco teratogênico de retinoides em uso sistêmico, precisam declarar e comprovar que não estão gravidas e, na maioria dos casos, requerem o uso de anticoncepcionais, independente de outra prática contraceptiva informada. Neste estudo, observou-se o uso de anticoncepcionais por mais de setenta por cento das pacientes por estaremna faixa etária de idade fértil, seguindo a classificação do Instituto Brasileiro de Geografia e Estatística (IBGE, 2020).

Referindo-se a capacidade de entendimento das NRR consultadas, a obrigação da legibilidade de receitas vem através da Lei Federal n 5.991 de 1973 na qual institui em seu artigo 35 que a receita somente deverá ser aviada se estiver legível, o que também determina oCódigo de Ética Médico em seu artigo 11, no qual é vedado ao médico receitar, atestar ou emitir laudos de forma secreta e ilegível (CFM, 2010).

De acordo com o CFM, acredita-se que não exista justificativa para tal conduta, que potencialmente pode trazer envolvimento e complicações gravíssimas e impactantes à vida do paciente como também a do profissional prescritor. A prescrição deve conter precisa e claramente o nome dos medicamentos, posologia, 
dose e qualquer que seja outra informação para que a receita seja entendida pelo paciente e pelo farmacêutico, o qual dispensará os medicamentos.

Esta obrigação também é estendida aos farmacêuticos para procederem a dispensação dos medicamentos, devendo verificar o cumprimento destas normas citadas acima para quaisquer tipos de prescrições medicamentosas e, diminuir ao máximo, possíveis de erros de medicação no âmbito da dispensação. O Conselho Federal de Farmácia comunga da mesma responsabilidade orientando aos farmacêuticos a zelarem pelo uso racional de medicamentos (CFF, 2001; CFF, 2014).

Em estudo realizado por Silvério e Leite (2010), foram encontradas notificações de receita ilegíveis em 36\% ( $n=82)$ dos documentos avaliados, sendo um número bem superior aoverificado nesta pesquisa. Já no estudo de Rosa et al. (2003), encontraram que $53,3 \%$ estavam legíveis, $24,5 \%$ pouco legíveis e $22,2 \%$ ilegíveis, assemelhando-se ao encontrado quanto as NRclassificadas como legíveis, que foram a maioria.

\section{CONCLUSÃO}

Foi possível observar que a falta de informações concretas na prescrição pode dificultaro tratamento do paciente, possibilitando erros de medicação, já que esta é a primeira etapa no ciclo de utilização dos medicamentos.

Mesmo com as orientações legais quanto a legibilidade de prescrições medicamentosas, foram encontradas informações nas notificações de receitas classificadas como ilegíveis, sendonecessário instituir medidas de sensibilização dos prescritores sobre a importância de uma prescrição clara, completa e precisa e, também, dos farmacêuticos responsáveis pela etapa de dispensação, para não procederem o serviço com quaisquer dúvidas oriundas da prescrição.

Percebesse a necessidade de educação sanitária contínua, fomentando práticas que diminuam erros potenciais e que dificultem a assistência integral à saúde dos usuários de medicamentos. Quantos aos erros de prescrição, estes 
podem ser reduzidos com a utilização de editores de textos para a prescrição, adoção de prescrições eletrônicas ou, mais simples, as prescrições impressas.

\section{REFERÊNCIAS BIBLIOGRÁFICAS}

BRASIL. Presidência da República. Lei n 5.991 de 17 de dezembro de 1973. Brasília: Casa Civil, 1973.

Constituição da República Federativa do Brasil de 1988. Brasília: DF, 1988.

. Lei $n^{\circ} 8078$, de 11 de setembro de 1990. Dispõe sobre a proteção do consumidor e dá outras providências. Diário Oficial da União. República Federativa do Brasil, Brasília: DF, 1990.

. Ministério da Saúde. Secretaria de Vigilância Sanitária. Portaria $n^{\circ} 344$ de 12 demaio de 1998. Brasília: DF, SVS, 1998.

. Ministério da Saúde. Secretaria de Vigilância Sanitária. Portaria nº6 de 29 dejaneiro de 1999. Brasília: DF, SVS, 1999.

Ministério da Saúde. Secretaria de Atenção à Saúde. Consulta Pública $n^{\circ} 10$, de 7 de dezembro de 2009. Brasília: DF. 2009.

Ministério da Saúde. Secretaria de Vigilância Sanitária. Portaria $n^{\circ} 1.159$ de 18 de novembro de 2015. Aprova o Protocolo de uso da isotretinoína no tratamento da acne grave. Brasília: DF, 2015. Civil, 2018.

Presidência da República. Lei n 13.732 de 08 de novembro de 2018. Brasília: DF,Casa

CONSELHO FEDERAL DE FARMÁCIA. RDC 357, de 20 de abril de 2001. Aprova o Regulamento Técnico de Boas Práticas de Farmácia. Brasília: CFF, 2001.

CONSELHO FEDERAL DE FARMÁCIA. Código de Ética Farmacêutica. RDC 596, de 21 de fevereiro de 2014. Dispõe sobre o Código de Ética Farmacêutica, o Código de Processo Ético e estabelece as infrações e as regras de aplicação das sanções disciplinares. Brasília: CFF, 2014.

CONSELHO FEDERAL DE MEDICINA. Código de Ética Médica. RDC CFM 1931, de 17 de setembro de 2009. Brasília: CFM, 2010.

INSTITUTO BRASILEIRO DE GEOGRAFIA E ESTATÍSTICA. Nupcialidade e Fecundidade. Brasília: IBGE, 2020.

ROSA, M. B.; NEIVA, H. M; ANACLETO, T. A; MENDES, D. P; FREITAS, F. O; LAGE, J. B; PERINI, E. Legibilidade de prescrições médicas com medicamentos potencialmenteperigosos em um hospital de Belo Horizonte, MG. Rev. BRAFH. v. 2, p. 22-8, 2003.

ROSA, M. B.; PERINI, E.; NACLETO, T. A.; NEIVA, H. M.; BOGUTCHI, T. Erros na prescrição hospitalar de medicamentos potencialmente perigosos. Rev Saúde Pública, v. 43, p.490-98, 2009. 
SILVÉRIO, M. S.; LEITE, I. C. G. Qualidade das prescrições em município de Minas Gerais:uma abordagem farmacoepidemiológica. Rev. Assoc. Med. Bras. v. 56, n. 6, p. 675-80, 2010.

VALLERAND, I. A.; LEWINSON, R. T.; FARRIS, M. S.; SIBLEY, C. D.; RAMIEN, M. L.; BULLOCH, A. G. M.; PATTEN, S. B. Efficacy and adverse events of oral isotretinoin foracne: a systematic review. British Journal of Dermatology. v. 178, p.76 -85, 2018.

ZANE, L. T. et al. A population-based analysis of laboratory abnormalities during isotretinoin therapy for acne vulgaris. Archives of dermatology, v. 142, n. 8, p. 1016-1022, 2006. 